\title{
Acute Kidney Injury in Dengue - Not Unprecedented
}

\author{
Javed Ismail ${ }^{1}$. Jhuma Sankar ${ }^{2}$ (ID \\ Received: 5 October 2020 / Accepted: 5 October 2020 / Published online: 16 October 2020 \\ (C) Dr. K C Chaudhuri Foundation 2020
}

The global burden of dengue is on an increasing trend since the past two decades with $70 \%$ of the total cases attributed to Asia. The reported number of cases according to the World Health Organization has increased from 2.4 million in 2010 to 4.2 million in 2019. In India, disease outbreaks occur predominantly in urban areas, but is now spreading rapidly to the rural population. The estimated prevalence of cases in India based on a sero-prevalence study is about 13 million in 2017 [1].

Critically ill children are prone to developing acute kidney injury (AKI) due to several reasons. AKI in dengue is a result of complex interplay of various factors like 1) cytopathic effects of the virus, 2) immune dysregulation, immune complex deposition, 3) hemolysis or rhabdomyolysis, 4) thrombotic microangiopathy, 5) as a part of multiorgan dysfunction due to macro and micro circulatory impairments and 6) nephrotoxic medications and hyperchloremia due to intravenous fluids. In a multicentre prospective cohort of 4683 critically ill children, incidence of AKI was $26.9 \%$ (95\% CI, 25.6 to 28.2) and of severe AKI was $11.6 \%$ (95\% CI, 10.7, 12.5) [2]. Studies on dengue report a varying incidence of AKI ranging from 0.2 to $35.7 \%$ [3]. In the retrospective cohort of children with dengue by Rajan et al. [4] published in this issue of the journal, the incidence of AKI was 20.5\% (26 out of 127 children). The risk of AKI increases with the severity of dengue and is associated with other organ dysfunction like liver, respiratory failure and severe bleeding $[5,6]$. In the index study, AKI was reported in $11.8 \%$ in dengue with warning signs and $28.6 \%$ in those with severe dengue [4]. AKI is an independent predictor of mortality in critically ill children with a risk of $11 \%$ in those with severe AKI vs. $2.5 \%$ in those without AKI [2]. The cohort of dengue follows the same pattern with increased risk of mortality in those with AKI $[5,7,8]$.

Jhuma Sankar

jhumaji@gmail.com

1 PICU, NMC Royal Hospital, Khalifa City, Abu Dhabi, UAE

2 Department of Pediatrics, All India Institute of Medical Sciences, New Delhi, India
The index study by Rajan et al. [4], reiterates the increased risk of AKI and its complications in the cohort of children with dengue. However, few limitations need mention. AKI in the index study was defined using the 'creatinine criteria' of Kidney Disease Improving Global Outcome (KDIGO) guidelines. However, recent reports suggest that nearly one-fifth of critically ill children with AKI may not experience an elevation of creatinine [9]. Also, patients identified by urine output criteria have a comparable poor outcomes like those by creatinine criteria [9]. This is echoed by an increase in relative risk of renal replacement therapy from 9 (95\% CI 3.9-21) in creatinine criteria to 28 (95\% CI 11.8-68) in urine output criteria and 166 (95\% CI 86-318) in those with both [9]. The risk of mortality was five times higher in those with both urine output and creatinine criteria compared to either of these alone [9]. Drawbacks of creatinine based diagnosis are influenced by factors like reduced muscle mass and fluid overload. Moreover, the creatinine starts rising only when substantial number of nephrons are already affected. Hence, the urine output criteria should always be used in combination with creatinine values to define AKI. Nevertheless, there is a group of 'subclinical AKI' where the glomerular filtration rate (GFR) may be normal with an ongoing tubular damage and dysfunction. This group goes undetected despite using both urine output and creatinine criteria and needs tubular damage biomarkers to identify them. The management of AKI remains the same in the dengue cohort which includes fluid management, modification of drug doses for GFR, avoidance of nephrotoxic medications, monitoring of electrolytes, and timely institution of renal replacement therapies. 


\section{Compliance with Ethical Standards}

Conflict of Interest None.

\section{References}

1. Murhekar MV, Kamaraj P, Kumar MS, et al. Burden of dengue infection in India, 2017: a cross-sectional population based serosurvey. Lancet Glob Health. 2019;7:e1065-73.

2. Kaddourah A, Basu RK, Bagshaw SM, Goldstein SL. Epidemiology of acute kidney injury in critically ill children and young adults. $\mathrm{N}$ Engl J Med. 2017;376:11-20.

3. Vachvanichsanong P, Thisyakorn U, Thisyakorn C. Dengue hemorrhagic fever and the kidney. Arch Virol. 2016;161:771-8.

4. Rajan M, Geminiganesan S, Sankaranarayanan S, Padmanaban R, Selvam MP. Renal manifestations in children with dengue fever hospitalized in pediatric intensive care unit. Indian J Pediatr [Internet]. 2020. Available at: http://link.springer.com/10.1007/ s12098-020-03402-z. Accessed 24 Sept 2020.
5. Laoprasopwattana K, Pruekprasert P, Dissaneewate P, Geater A, Vachvanichsanong P. Outcome of dengue hemorrhagic fevercaused acute kidney injury in Thai children. J Pediatr. 2010;157: 303-9.

6. Sultana A, Rumana J, Roy S, et al. Renal involvement in children with dengue fever: a study in tertiary care hospital of Bangladesh. Int J Nephrol. 2020;2020:1-6.

7. Kuo M-C, Lu P-L, Chang J-M, et al. Impact of renal failure on the outcome of dengue viral infection. Clin J Am Soc Nephrol. 2008;3: 1350-6.

8. Poddar S, Sharma S, Kaur C, Chellani HK. Acute kidney injury in dengue among hospitalized children: a prospective view. Saudi J Kidney Dis Transplant. 2020;31:407-14.

9. Kaddourah A, Basu RK, Goldstein SL, Sutherland SM. Oliguria and acute kidney injury in critically ill children: implications for diagnosis and outcomes. Pediatr Crit Care Med. 2019;20:332-9.

Publisher's Note Springer Nature remains neutral with regard to jurisdictional claims in published maps and institutional affiliations. 